\title{
Antisense Oligonucleotide In Vitro Protein Binding Determination in Plasma, Brain and Cerebral Spinal Fluid Using Hybridization LC-MS/MS
}

\author{
Guilherme Guimaraes, Long Yuan, and Pei Li
}

Drug Metabolism and Pharmacokinetics, Biogen, Cambridge, Massachusetts 02142, United

States (G.G., L.Y., P.L.) 
Running Title: In Vitro ASO Protein Binding Using Hybridization LC-MS/MS

\section{Corresponding Author}

Long Yuan

Drug Metabolism and Pharmacokinetics

Biogen, 225 Binney Street, Cambridge, Massachusetts 02142, United States

Email: long.yuan@biogen.com

Number of text pages: 26

Number of tables: 3

Number of figures: 5

Number of references: 49

Number of words in Abstract: 192

Number of words in Introduction: 750

Number of words in Discussion: 1370

\section{Abbreviations}

ACSF, artificial cerebral spinal fluid

ASO, antisense oligonucleotide

CNS, central nervous system

CSF, cerebral spinal fluid

$f_{\mathrm{u}}$, unbound fraction

LC-MS/MS, liquid chromatography-tandem mass spectrometry

MOE, 2'-O-(2-methoxyethyl)-oligoribonucleotides 
MW, molecular weight

MWCO, molecular weight cutoff

MRM, Multiple reaction monitoring

NSB, nonspecific binding

PBS, phosphate-buffered saline

PCR, polymerase chain reaction

PD, pharmacodynamic

PK, pharmacokinetic

RED, rapid equilibrium dialysis 


\begin{abstract}
The development of quantitative models for prediction of drug pharmacokinetics based on in vitro data has transformed early drug discovery. Drug unbound fraction $\left(f_{\mathrm{u}}\right)$ characterization is a key consideration in pharmacokinetic (PK) and pharmacodynamic (PD) modeling, assuming only unbound drug can interact with the target, and therefore has direct implications in the efficacy and potential toxicity of the drug. The current study describes the implementation of a hybridization LC-MS/MS platform for the direct quantitation of antisense oligonucleotide (ASO) $f_{\mathrm{u}}$. The method provides substantial improvements including minimal matrix effects and high specificity when compared to previously used oligonucleotide $f_{\mathrm{u}}$ detection methods such as ligand binding assays or liquid scintillation. The hybridization LC-MS/MS platform was integrated with ultracentrifugation, ultrafiltration and equilibrium dialysis, and method performance for each technique was evaluated. While ASO protein binding has been previously characterized in plasma, there were no studies that quantitated ASO $f_{\mathrm{u}}$ in brain or CSF. As ASOs continue to undergo clinical trials for neurological and neuromuscular indications, $f_{\mathrm{u}}$ characterization in brain and CSF can provide invaluable information about ASO distribution and target engagement in the central nervous system, therefore providing support for in vivo PKPD data characterization.
\end{abstract}

\title{
Significance Statement
}

A novel hybridization LC-MS/MS based approach was successfully developed for the determination of ASO in vitro protein binding in plasma, and for the first-time brain and CSF. Ultrafiltration, equilibrium dialysis, and ultracentrifugation were assessed for the separation of unbound ASO from biological matrices. The hybridization LC-MS/MS platform provided unique 
advantages, including minimal matrix effects and high specificity, comparing to traditional ligand binding assays or liquid scintillation approaches, which enabled efficient and reliable in vitro protein binding assay. 


\section{Introduction}

Antisense oligonucleotides (ASOs), defined as short synthetic oligonucleotides with single-stranded sequences complementary to certain mRNA sites, have been under drug development for approximately 30 years, with the first FDA approved drug, Fomivirsen, being approved in 1998 (Stein and Castanotto, 2017). Chemical modifications such as substitution of one non-bridging oxygen (phosphate) for a sulfur (phosphorothioate) have increased stability against nuclease degradation as well as plasma protein binding, with phosphorothioated oligonucleotides being highly protein bound (>85\%) (Levin et al., 2007; Geary et al., 2015). Proper characterization of ASOs chemical modifications and its biological ramifications are important since ASO pharmacokinetic properties are largely driven by chemistry rather than sequence (Geary, 2001; Geary et al., 2015).

Drug unbound fraction $\left(f_{\mathrm{u}}\right)$ quantification in any given matrix is essential in understanding drug pharmacokinetics since it is generally accepted that only the unbound drug is available and responsible for drug efficacy and potential drug toxicity (Rowland et al., 2011; Mariappan et al., 2013; Roberts et al., 2013). $f_{\mathrm{u}}$ quantification provides valuable insight into drug absorption, distribution, metabolism, excretion, and toxicity. While ASO $f_{\mathrm{u}}$ has been previously characterized in plasma, ASO $f_{\mathrm{u}}$ in matrixes of high interest such as brain and cerebral spinal fluid (CSF) is still unknown. Due to relatively large molecular size and multiple negative charges, ASOs do not efficiently cross the blood brain barrier (Geary et al., 2015; Bennett et al., 2017). However, this limitation has been mitigated through intracerebroventricular or intrathecal delivery while less invasive delivery techniques continue to be developed (Miller et al., 2013; Wurster and Ludolph, 2018; Alarcón-Arís et al., 2020; Min et al., 2020). To better 
understand ASO exposure and target occupancy in the CNS, proper $f_{\mathrm{u}}$ characterization in brain and CSF is crucial.

Historically, $f_{\mathrm{u}}$ determination for small-molecule drug candidates has been determined by ultrafiltration, ultracentrifugation, or equilibrium dialysis, which are techniques based on the physical separation of unbound drug molecules from those bound to proteins (Pacifici and Viani, 1992). In vitro drug $f_{\mathrm{u}}$ results are one of the properties used to prioritize certain drug candidates into further stages of drug development. Due to their physical properties such as relatively high molecular weight, linear structure, and nonspecific binding, ASOs present unique challenges in $f_{\mathrm{u}}$ determination through traditional techniques. Because of the linear conformation of ASOs, the molecular weight cutoff (MWCO) for dialytic membranes needs to be much higher than the ASO molecular weight (generally $\sim 7 \mathrm{KDa}$ ). The lack of commercially available equilibrium dialysis membranes with MWCO over 20K makes this technique incompatible with ASOs (Rocca et al., 2019). Ultrafiltration also requires a membrane medium, which becomes a major source for oligonucleotide nonspecific adsorption and therefore low recoveries. To overcome this issue, membrane pre-treatment with surfactants or sacrificial oligonucleotides that are irrelevant to the analyte have shown to provide acceptable recoveries (Watanabe et al., 2006; Humphreys et al., 2019). Ultracentrifugation bypasses the need for a membrane, however low recoveries have been reported for siRNA, potentially due to differential sedimentation of macromolecules (Hughes et al., 1938; Humphreys et al., 2019). Alternatively, electrophoretic mobility shift has been evaluated as a method for determining siRNA $f_{\mathrm{u}}$ in plasma (Rocca et al., 2019). While this technique also bypasses the need for a membrane, limited sample throughput prevents it from being widely applied in drug discovery. 
Previous detection methods for oligonucleotide $f_{\mathrm{u}}$ determination included liquid scintillation and hybridization techniques such as real-time polymerase chain reaction (PCR). There are several advantages when choosing LC-MS/MS over previous methods, including the generation of more quantitative values, easier normalization, and less biased results coming from different variations of PCR techniques (Wang et al., 2013; Basiri et al., 2019). Additionally, liquid scintillation requires the synthesis of radiolabeled ASOs that are expensive to produce and challenging to dispose of.

In this work, ASO $f_{\mathrm{u}}$ in plasma, brain and CSF were characterized through an ultrafiltration hybridization LC-MS/MS platform. This is the first time LC-MS was employed for direct quantitation of ASO $f_{\mathrm{u}}$ in not only plasma, but also brain tissue and CSF, for a more complete understanding of ASO protein binding. The method allowed specific and sensitive analysis, wide linear range, and automation of the hybridization extraction (Li et al., 2020). Complete elimination of matrix effects enabled the direct comparison between unbound and bound ASO in different sample compositions, which was otherwise unachievable by previous methods. In addition to ultrafiltration, equilibrium dialysis and ultracentrifugation were also evaluated, although membrane incompatibility, limited sample throughput, and sampling bias hinders their performance. $f_{\mathrm{u}}$ was characterized for three different ASOs across different matrixes in multiple species.

\section{Materials and Methods}

\section{Materials}

Analyte ASO-1, ASO-2, ASO-3, and the internal standard (ASO-4) were proprietary assets of Biogen and provided by its collaborator, with basic information shown in Table 1. 
Capture probes (biotinylated reverse complimentary DNA) were synthesized by Integrated DNA Technologies (Coralville, IA). Rapid equilibrium dialysis (RED) device [12 kDa MWCO], Pierce $^{\mathrm{TM}}$ BCA protein assay kit, Dynabeads MyOne streptavidin C1 and RNA grade proteinase K solution $(20 \mathrm{mg} / \mathrm{ml})$ were purchased from Thermo Fisher Scientific (Waltham, MA). Clarity OTX lysis-loading buffer was acquired from Phenomenex (Torrance, CA). Amicon Ultra 0.5-ml centrifugal filters [10, 30, 50 and $100 \mathrm{kDa}$ MWCO], Trizma base, sodium chloride, Tween 20 and ethylenediaminetetraacetic acid (EDTA) were acquired from MilliporeSigma (St. Louis, MO). 1,1,1,3,3,3-Hexafluoro-2-methyl-2-propanol (HFMIP) was obtained from Alfa Aesar (Tewksbury, MA). N,N-dimethylcyclohexylamine (DMCHA) was obtained from TCI America (Portland, OR). HPLC grade acetonitrile (ACN) and DL-1,4-dithiothreitol (DTT) were obtained from Fisher Scientific (Hampton, NH). Pooled rat, mouse, dog, monkey and human plasma (K2EDTA) as well as male rate brain homogenate and monkey CSF were obtained from BioIVT (Westbury, NY). Artificial cerebrospinal fluid (ACSF) was acquired from Tocris Bioscience (Minneapolis, MN). Biogen in-house Milli-Q deionized water was used.

\section{Ultrafiltration}

A volume of $0.5 \mathrm{ml}$ phosphate-buffered saline (PBS; $0.137 \mathrm{M} \mathrm{NaCl}, 0.0027 \mathrm{M} \mathrm{K}$, 0.0119M Phosphates) with $0.1 \%$ Tween 20 (v/v; PBST) was added to Amicon Ultra 0.5-ml centrifugal three times to remove residual glycerin and condition filter. Following every PBST addition, 30, 50 and $100 \mathrm{kDa}$ filters were spun in a bench-top centrifuge for $10 \mathrm{~min}$ at $3000 \times \mathrm{g}$ while $10 \mathrm{kDa}$ filters were spun at $5000 \times \mathrm{g}$. After the third addition of PBST, filters were incubated at room temperature for $15 \mathrm{~min}$ before centrifugation. Any residual treatment solution was removed, while the collection tube was retained. For the sample treatment experiment, four 
conditions were evaluated: $0.1 \%$ Tween-20 in PBS, $5 \mu \mathrm{M}$ sacrificial ASO in PBS, $5 \mu \mathrm{M}$ sacrificial ASO in PBS with $1 \%$ Tween-20, and $5 \mu \mathrm{M}$ sacrificial ASO in PBS with $0.1 \%$ Tween20. Spiked matrixes were added to filters immediately after treatment to ensure filters never dried out. For recovery experiments, $1 \mu \mathrm{M}$ ASO-1, $1 \mu \mathrm{M}$ warfarin and $1 \mu \mathrm{M}$ antipyrine were cospiked into PBS. A volume of $0.5 \mathrm{ml}$ was transferred to treated filters and incubated at room temperature for $15 \mathrm{~min}$. Following incubation, $10 \mathrm{kDa}$ MWCO filters were centrifuged at $3000 \times$ $\mathrm{g}$ for $1 \mathrm{~min}$ while 30,50 and $100 \mathrm{kDa}$ filters were centrifuged at $1500 \times \mathrm{g}$. Centrifugation time varied for different matrixes and filter MWCO sizes.

Plasma (pre-equilibrated to $37^{\circ} \mathrm{C}$ ) was co-spiked with $1 \mu \mathrm{M}$ ASO, $1 \mu \mathrm{M}$ warfarin and 1 $\mu \mathrm{M}$ antipyrine and incubated at $37{ }^{\circ} \mathrm{C}$ while shaking at $500 \mathrm{rpm} .500 \mu \mathrm{l}$ of spiked plasma was transferred to treated filters and $10 \mathrm{kDa}$ MWCO filter centrifuged at $3000 \times \mathrm{g}$ for 2 min, while 30,50 , and $100 \mathrm{kDa}$ centrifuged at $1500 \times \mathrm{g}$ for the same amount of time. Same procedure was repeated for brain homogenate and CSF samples, but centrifugation time varied between matrixes: brain homogenate $-10 \mathrm{kDa}(3000 \times \mathrm{g}), 30,50$, and $100 \mathrm{kDa}(1500 \times \mathrm{g})$ for $3 \mathrm{~min}, \mathrm{CSF}$ - $10 \mathrm{kDa}(3000 \times \mathrm{g}), 30,50$, and $100 \mathrm{kDa}(1500 \times \mathrm{g})$ for $2 \mathrm{~min}$. Centrifugation time and speed were optimized to ensure sufficient but no more than $20 \%$ of the sample crossed the filter. CSF was also co-spiked with $1 \mu \mathrm{M}$ ASO, $1 \mu \mathrm{M}$ warfarin and $1 \mu \mathrm{M}$ antipyrine, while brain homogenate was co-spiked with $1 \mu \mathrm{M}$ ASO and $1 \mu \mathrm{M}$ verapamil. Ultracentrifugation and rapid equilibrium dialysis methods, as well as the small-molecule LC-MS/MS quantification method are provided in the Supplemental Section.

\section{ASO Quantification}


For ASO quantification, $10 \mu \mathrm{l}$ of the filtered (representing "free") or unfiltered (representing "total") sample was combined with $90 \mu \mathrm{l}$ of corresponding blank matrix in a Kingfisher 96 deep-well plate (Thermo Fisher Scientific, Waltham, MA). Sample preparation and LC-MS/MS analysis were conducted following a previously published protocol from Li and co-workers with minor modifications ( $\mathrm{Li}$ et al., 2020). Multiple reaction monitoring (MRM) transitions used were: ASO-1 $(785 \rightarrow 94.9 \mathrm{~m} / \mathrm{z})$, ASO-2 $(637.7 \rightarrow 94.9 \mathrm{~m} / \mathrm{z})$, ASO-3 $(660.1 \rightarrow 94.9$ $\mathrm{m} / \mathrm{z})$.

Recovery and $f_{\mathrm{u}}$ were calculated as previously described by Humphreys and co-workers using the following equations:

$$
\begin{aligned}
\% \text { Recovery }(\text { buffer }) & =\frac{[\text { receiver }]}{[\text { donor }]} \times 100 \\
f_{u} & =\frac{[\text { receiver }]}{[\text { donor }]}
\end{aligned}
$$

where [donor] is the original concentration of drug in the spiked matrix prior to addition to filtration apparatus, and [receiver] is the drug concentration in the ultrafiltrate (Humphreys et al., 2019).

In this study $f_{\mathrm{u}}$ is shown in terms of percentage, meaning that equation 2 is multiplied by 100 . Lastly, the dilution for brain homogenate (1:8) was adjusted through the following equation described by Kalvass and co-workers (Kalvass and Maurer, 2002; Kalvass et al., 2007):

$$
\text { undiluted } f_{u}=\frac{1 / D}{\left(\left(1 / f_{u, \text { measured }}\right)-1\right)+1 / D}
$$


D represents the dilution factor of brain tissue and $f_{\mathrm{u} \text {,measured }}$ is the ratio described in eq.2. All ultrafiltration and RED experiments had a minimum number of 3 replicates $(\mathrm{N} \geq 3)$. Due to limited throughput, ultracentrifugation experiments were not replicated $(\mathrm{N}=1)$.

\section{Results}

\section{Recovery Evaluation}

To assess analyte nonspecific binding (NSB) to the filter membrane, the major potential limitation of ultrafiltration, recovery was evaluated for different filter pre-treatment conditions and different filter sizes by measuring the percentage of drug remaining in a protein free medium after filtration (Toma et al., 2021). Recovery determination was calculated through Eq.1. Figure 1A shows the recovery of $1 \mu \mathrm{M}$ ASO-1 in PBS under five different filter treatment conditions including untreated (filter rinsed with PBS), 0.1\% Tween-20 in PBS, $5 \mu \mathrm{M}$ sacrificial ASO in PBS, $5 \mu \mathrm{M}$ sacrificial ASO in PBS with $1 \%$ Tween-20, and $5 \mu \mathrm{M}$ sacrificial ASO in PBS with 0.1\% Tween-20. Recovery was assessed in 30K MWCO filters, which was the size showing significant but mitigatable loss to NSB. Consistent with previous reports, filter pre-treatment with either Tween-20 or sacrificial ASO was shown to minimize NSB (Watanabe et al., 2006; Humphreys et al., 2019). Based on recovery against a commonly accepted criterium at $80 \%$, $0.1 \%$ and higher concentrations of Tween-20, with or without the combination of sacrificial ASO, provided sufficient anti-NSB protection, suggesting $0.1 \%$ Tween-20 to be the ideal blocking agent. Previous studies have shown Tween-20 to be the best blocking agent over several conditions including heparin, irrelevant oligonucleotides, bovine serum albumin, and others (Humphreys et al., 2019; Rocca et al., 2019). 
Upon determining optimal filter treatment with $0.1 \%$ Tween-20, recovery of $1 \mu \mathrm{M}$ ASO1 co-spiked with $1 \mu \mathrm{M}$ warfarin and antipyrine was evaluated at four different filter MWCO sizes (Figure 1B and 1C). While low recovery was observed for $10 \mathrm{~K}$ filters, acceptable recovery was achieved for 30,50, and 100K MWCO filters, suggesting a correlation between recovery and MWCO and further suggesting that unbound ASO cannot effectively pass $10 \mathrm{~K}$ filter due to size constraint. MWCO of membranes is determined based upon a relationship between molecular weight and globular size as defined by Humphreys and co-workers (Humphreys et al., 2019). Given this relationship, the linear nature of oligonucleotides requires much higher MWCO, even though the MW of ASOs in this study was below $10 \mathrm{kDa}$ (Humphreys et al., 2019). While filter size and treatment have direct implications on ASO recovery, these variables have no apparent impact on the recovery of small molecule controls (Figure 1C), confirming that the filter size had no impact on drug molecules that were significantly smaller than the MWCO. In summary, conditions of MWCO above $30 \mathrm{~K}$ and treatment with $0.1 \%$ Tween-20 were considered sufficient in ensuring unbound ASO recovery. Recovery of $83 \%$ was observed at $30 \mathrm{~K}$ MWCO in comparison to $96 \%$ recovery at $50 \mathrm{~K}$ and $100 \mathrm{~K} \mathrm{MWCO}$.

\section{ASO $f_{\mathrm{u}}$ Determination in Plasma}

Plasma $f_{\mathrm{u}}$ experiments were conducted at all filter sizes (Figure 2A), and experiments were orthogonally validated through co-spiked small molecules that are either highly protein bound (warfarin) or highly protein unbound (antipyrine). As filter MWCO increased, measured plasma $f_{\mathrm{u}}$ also increased for both ASO and small molecule controls. The upward trend suggests protein bleeding, specifically for proteins carrying bound drug molecules, in larger MWCO filter sizes as the responsible factor for artificially higher plasma $f_{\mathrm{u}}$ measurements. In the context of 
this study, protein bleeding is defined as proteins with higher MW than filter cutoff or proteins carrying bound drug molecules that pass through the filter and end up in the ultrafiltrate. Previous studies have shown ASOs to be highly bound to albumin (Cossum et al., 1993; Srinivasan et al., 1995; Watanabe et al., 2006). Albumin has a relatively low MW ( 66.5 kDa) and has been identified in the ultrafiltrate of a 50K MWCO ultrafiltration assay through electrophoretic mobility, suggesting protein bleeding even though the MW is higher than the MWCO (Humphreys et al., 2019). Similarly, warfarin is also highly bound to albumin and its concentration in the ultrafiltrate is also artificially higher when using $50 \mathrm{~K}$ or higher MWCO filters, which results in a similar upward trend to those observed in Figure 2A (Supplemental Figure 1A). In contrast, minimal change in antipyrine concentration in the ultrafiltrate was observed in different MWCO filters, since it is highly unbound and not significantly affected by protein bleeding (Supplemental Figure 1B). To minimize the impact of protein bleeding, the smallest filter size that still achieved satisfactory recovery, in this case $30 \mathrm{~K}$ MWCO, was chosen as the final condition. Plasma $f_{\mathrm{u}}$ measurements were repeated in two other ASOs across 5 species (Figure 2B). Interspecies differences in plasma $f_{\mathrm{u}}$ were observed following a trend in the order of rat $<$ monkey $<$ human $\approx \operatorname{dog}<$ mouse, which was consistent with previously reported results (Watanabe et al., 2006). Additionally, Watanable and co-workers described an inverse relationship between ASO sequence length and unbound concentration through plasma $f_{\mathrm{u}}$ measurements of 10 shortmer metabolites. While plasma $f_{\mathrm{u}}$ for ASO metabolites was not evaluated in this study, higher unbound concentrations were observed for ASO-3 (17mer) in comparison to the other 2 ASOs (20mers). The $30 \mathrm{kDa}$ MWCO ultrafiltration method was orthogonally validated through previously characterized small-molecule controls, shown in Table 2. The results generated from the ultrafiltration method were consistent with those from standard 
RED methods, as well as the literature reported values. This further demonstrated the validity of the developed ultrafiltration method.

\section{ASO $f_{u}$ Determination in Brain Tissue Homogenate}

Ultrafiltration experiments were similarly conducted in rat brain tissue homogenate. ASO $f_{\mathrm{u}}$ determination at $1 \mu \mathrm{M}$ was orthogonally validated through co-spiked $1 \mu \mathrm{M}$ verapamil, a better characterized compound for brain $f_{\mathrm{u}}$. Brain $f_{\mathrm{u}}$ determination was carried out at four different MWCO sizes, across 3 different ASOs. As seen in Figure 3, similar upward trend in measured brain $f_{\mathrm{u}}$ was observed as filter sizes increased, suggesting protein bleeding to influence measured brain $f_{\mathrm{u}}$. Just like in plasma samples, the most suitable filter size was $30 \mathrm{kDa}$ MWCO. Overall, rat brain $f_{\mathrm{u}}$ was 7 -fold lower in comparison to rat plasma $f_{\mathrm{u}}$. Differences in $f_{\mathrm{u}}$ in brain homogenate in comparison to plasma $f_{\mathrm{u}}$ are likely due to differences in protein concentration in the two matrices. The correlation between higher protein content and higher protein binding has been discussed by Liu and co-workers (Liu et al., 2014). Based on this correlation, lower brain $f_{\mathrm{u}}$ is likely due to brain's higher protein content in comparison to protein content in plasma.

\section{ASO $f_{u}$ in Cerebral Spinal Fluid}

The final biological matrix in which ASO $f_{\mathrm{u}}$ was characterized was monkey CSF. As expected, CSF $f_{\mathrm{u}}$ was significantly higher in comparison to other biological matrices due to its relatively low protein composition. Initially, protein binding experiments were carried out in CSF and protein-free artificial CSF (ACSF). Figure 4A showed ASO-1 $f_{\mathrm{u}}$ in CSF and ACSF in different filter sizes. ACSF protein binding results were similar to recovery experiments, since both ACSF and PBS are protein-free. Differences between $30 \mathrm{~K}$ and $50 \mathrm{~K}$ filters in CSF were proportional to 
differences between these sizes in ACSF. Furthermore, 30K CSF $f_{\mathrm{u}}$ values can be corrected in accordance to differences in recovery between $30 \mathrm{~K}$ and $50 \mathrm{~K}$ filters in ACSF. The $f_{\mathrm{u}}$ corrected is similar to $f_{\mathrm{u}}$ in $50 \mathrm{~K}$ filters (Supplemental Figure 2), suggesting that protein bleeding is less concerning in CSF, likely due to its relative low protein composition. Overall, these results suggested that $50 \mathrm{~K}$ filters were the appropriate size for CSF $f_{\mathrm{u}}$ quantification without the need for recovery normalization. CSF $f_{\mathrm{u}}$ for different ASOs was quantified in 50K MWCO filters (Figure 4B). Co-spiked warfarin and antipyrine CSF and ACSF $f_{\mathrm{u}}$ is available in supplemental figure 3. Similar to warfarin $f_{\mathrm{u}}$ in plasma and brain homogenate, there is an upward trend in CSF $f_{\mathrm{u}}$ as the filter MWCO increases, which can be attributed to protein bleeding. However, warfarin CSF $f_{\mathrm{u}}$ measurements were not significantly different between $30 \mathrm{~K}$ and $50 \mathrm{~K}$ MWCO filters, due to the low concentrations of CSF proteins within this narrow MW range, and therefore limited impact from protein bleeding. For antipyrine, due to its highly unbound nature, filter size had no impact on CSF $f_{\mathrm{u}}$ measurements.

\section{Equilibrium Dialysis}

Rapid equilibrium dialysis (RED) is a popular technique for high throughput protein binding assays and often referred to as the "gold standard". (Trainor, 2007) As previously shown, filter MWCO plays a key role in ASO recovery. The first step in RED was to confirm if ASO concentrations both in the sample compartment and solvent compartment reached equilibrium in a protein free medium. Despite different membrane pre-treatments and prolonged incubations, system equilibrium was never reached with the biggest available MWCO at $12 \mathrm{~K}$, suggesting ASOs were not physically able to cross the membrane limited by the pore size. Results were also comparable to poor ASO permeability of similar $\mathrm{MWCO}$ at $10 \mathrm{~K}$ demonstrated in the 
ultrafiltration section. Both small molecule controls (warfarin and antipyrine) reached equilibrium under the assessed experimental conditions (Figure 5), which not only orthogonally confirmed the validity of the experiment, but also pointed the loss of ASO to its higher MW and unique NSB.

\section{Ultracentrifugation}

In addition to ultrafiltration and RED, an ultracentrifugation method was explored and its compatibility with ASOs was assessed. Polycarbonate tubes were filled with either $1 \mathrm{ml}$ of rat plasma or PBS, co-spiked with $1 \mu \mathrm{M}$ ASO-1 and $1 \mu \mathrm{M}$ warfarin. Spiked samples were centrifuged at 400,000 $\times \mathrm{g}$ for $1,2,3$ and 4 hours. Upon collection of each time point, $1 \mathrm{ml}$ sample was divided into 4 vertical quadrants of equal volumes $(250 \mu \mathrm{l}$ each). With the top layer (first $250 \mu \mathrm{l}$ ) being "Q1", second $250 \mu \mathrm{l}$ being "Q2" and so on. ASO-1 and warfarin concentrations were quantified for every quadrant, and their recoveries were calculated against pre-centrifuge samples and shown in Table 3. Protein concentration for different quadrants is shown in Supplemental Table 1.

Similar to ultrafiltration, analyte recovery was first assessed in PBS and confirmed that there was no significant loss of ASO-1 or warfarin to the experimental system without the impact of any protein binding. It was noticed that ASO-1 quickly formed a concentration gradient 74.3 $153.5 \%$ from Q1 to Q4 at 1 hour and maintained the similar gradient to the end, which was not observed in warfarin. Warfarin initially formed a concentration gradient in PBS (potentially due to poor solubility and insufficient mixing), but equilibrium between the quadrants was observed in hours 3 and 4 as expected for a small molecule. The concentration gradient observed for ASO suggested that the relatively higher MW of ASO compared to small molecules 
led to slight precipitation of unbound ASO at such strong ultracentrifugation conditions, though approximately $80 \%$ recovery might still be considered acceptable.

In plasma samples, significant vertical gradients were observed for both ASO-1 and warfarin, due to the precipitation of protein bound analytes as expected. After hours, the solution system started to reach equilibrium, in which Q1 was considered protein free as confirmed by total protein quantitated at approximately $0.4 \%$ of original. Therefore, ASO-1 rat plasma $f_{\mathrm{u}}$ was determined to be $2.0 \%$ based on the Q1 concentration at 4 hours, which was 2 -fold higher than the $f_{\mathrm{u}}$ determined through ultrafiltration $(0.89 \%)$. Warfarin plasma $f_{\mathrm{u}}$ was measured at $0.6 \%$, lower in comparison to ultrafiltration experiments $(1 \%)$ and literature (1.1\%). Such different directions of bias between ASO and warfarin made it difficult to interpret the results from a systematic perspective. Considering a few facts that 1) the experiment was not strictly validated by co-spiked warfarin, 2) unbound ASO also forms a concentration gradient under high centrifugal forces, 3) measured ASO $f_{\mathrm{u}}$ was higher than references, and 4) the procedure suffered from low throughput and therefore limited replicates $(n=1)$, the approach of ultracentrifugation was deemed impractical for ASO $f_{\mathrm{u}}$ measurement.

\section{Discussion}

The current study assessed ultrafiltration, ultracentrifugation and equilibrium dialysis to measure ASO $f_{\mathrm{u}}$ in plasma, brain homogenate, and CSF, while successfully implementing LCMS/MS as a sensitive and direct quantitation method. This is the first time LC-MS/MS has been employed for quantification of ASO in vitro protein binding and the first time ultracentrifugation and equilibrium dialysis are evaluated for such application. 
Hybridization LC-MS/MS integration. Both the hybridization extraction and LC-MS/MS quantification offer major advantages over previously published methods (Watanabe et al., 2006). On the detection side, MS provides high specificity and simple signal standardization through the implementation of an internal standard. However, one of the typical drawbacks when coupling MS to the analysis of biological samples is matrix effect, which is defined as the variations in the analyte signal caused by matrix. To minimize the impact of matrix effects, calibration curves are usually prepared in the same matrix as the study samples, which becomes an inefficient process as the number of matrix components grow. In the case of protein binding experiments, matrix interference becomes problematic when measuring separated unbound ASO and total ASO existing in matrices of different compositions (one protein free and the other protein rich), as none of previously available analytical methods was able to completely avoid matrix effects. The adopted hybridization protocol overcomes matrix effect limitations by capturing the desired ASO from the biological matrix, washing residual matrix through several washes, and finally eluting the analyte in neat solution containing internal standard. Matrix removal allows for cross-matrix quantification, therefore increasing sample preparation efficiency (Li et al., 2020). Due to the mechanism of hybridization and the need of a specific capture probe, the ultrafiltration-based hybridization LC-MS/MS method is suitable for accurate protein binding assessments of identified lead ASOs, instead of screening large numbers of ASOs as a high-throughput method. Multiple ASOs can be analyzed in a batched manner as long as separate capture probes are used. The hybridization LC-MS/MS platform is robust, and compatible with ultrafiltration, RED and ultracentrifugation experiments. 
Ultracentrifugation limitations. In theory, ultracentrifugation was a promising technique in ASO $f_{\mathrm{u}}$ quantification because it does not require a filter membrane, therefore avoiding ASO NSB to filter apparatus, ASO poor membrane permeability, and protein bleeding. Recently, low recovery, likely due to sedimentation, was reported in ultracentrifugation of siRNA (Humphreys et al., 2019). However, throughout the ultracentrifugation experiments we did not observe the same recovery loss, perhaps due to the lower molecular weight of ASOs (approximately $7 \mathrm{kDa}$ ) in comparison to siRNA (approximately $14 \mathrm{kDa}$ ). Even though appropriate recovery was observed in the final (4 hour) time point $(82.5 \%)$, variance from the recoveries in previous time points suggests sampling challenges. The special division of four quadrants was arbitrary and practically difficult by manual pipetting. Another major limitation with ultracentrifugation is sample throughput, as high-speed rotors have limited capacity. In this study, only 10 samples were able to be analyzed per run, largely limiting the number of replicates to reliably make measurements. Additionally, ultracentrifuges able to handle speeds over 400,000 $\times \mathrm{g}$ for multiple hours are not widely available. Because of lower sample throughput, and lower reproducibility, ultracentrifugation was a less ideal choice compared to ultrafiltration.

Ultrafiltration filter considerations. Due to their linear structure, ASOs require MWCO filters that are substantially higher than the ASO's MW. Humphreys et al. suggested through a siRNA crystal structure that hydrodynamic radius must be taken into consideration when choosing MWCO filter sizes for molecules of linear conformations (Humphreys et al., 2019). In their study, a 50K MWCO filter had to be used over 30K MWCO filters in order to achieve appropriate recovery for a GalNAc-conjugated siRNA. Watanable et. al. reports over $85 \%$ recovery when using 30K MWCO filters treated with $5 \mu \mathrm{M}$ irrelevant ASO (not radiolabeled) for 
the analysis of a 20mer phosphonothioate ASO (Watanabe et al., 2006). These results suggest that double stranded conformations are more rigid in comparison to single stranded conformations, and therefore smaller MWCO filters $(30 \mathrm{~K})$ are compatible with ASOs. When choosing between different MWCO sizes, the main consideration becomes ASO bound to small proteins crossing the filter membrane.

In this study, $f_{\mathrm{u}}$ was determined by Eq2 but in reality, protein bound ASOs that cross high MWCO membranes must be taken into consideration. A more realistically representation would be:

$f_{u}=\frac{[\text { receiver }]}{[\text { donor }]}=\frac{[\text { ASO unbound in ultrafiltrate }]+[\text { ASO protein bound in ultrafiltrate }]}{[\text { donor }]}$ Eq. 4

The choice of filter size should be the size at which [ASO protein bound in ultrafiltrate] is the least, while [ASO unbound in ultrafiltrate] is recovered as much as possible. For these reasons, 30K filters were chosen in the analysis of protein rich matrices (brain homogenate and plasma).

Interestingly, our results show that filter size is also matrix dependent. For protein rich matrixes such as brain homogenate and plasma, significant differences between $30 \mathrm{~K}$ and $50 \mathrm{~K}$ filters were observed, but in CSF (low protein concentration), differences between $30 \mathrm{~K}$ and $50 \mathrm{~K}$ filters were proportional to their differences in recovery. With this in mind, the filter size that was used for $f_{\mathrm{u}}$ determination in CSF was 50K MWCO, while $30 \mathrm{~K}$ might also be considered when corrected by recovery.

Throughout the ultrafiltration experiments we noticed occasional outliers likely resulting from defective filters or tube-to-tube variations from the manufacture. All the observed outliers were substantially higher than the average for both ASOs and small molecules. Outliers were 
removed using Pierce's criterion, which was selected as a pre-determined elimination criterion (Ross, 2003). A potential limitation from ultrafiltration experiments is that even when using $30 \mathrm{~K}$ MWCO filters, it is possible that ASO bound to smaller proteins or biomolecules may pass through the filter and end up in the ultrafiltrate, which is, however, an inevitable scenario for any protein binding assay based on the molecular weight difference between bound and unbound ASO.

Brain homogenate and CSF $\boldsymbol{f}_{\mathbf{u}}$. Development of quantitative models for prediction of drug absorption, distribution, and excretion has transformed early drug discovery. In vivo measurements in many instances are possible, but it requires expensive and complex techniques with low sample throughput, which limits its application in early drug discovery and highlights the importance of in vitro data based prediction models (Trainor, 2007). Total drug and unbound concentrations in various matrixes, including plasma and brain provide valuable information about drug pharmacokinetics.

The ratio of total drug concentration in the tissue to total concentration of drug in the plasma at steady state $\left(\mathrm{K}_{\mathrm{p}}\right)$, may be used to predict the extent of tissue distribution (Jones and Rowland-Yeo, 2013). $\mathrm{K}_{\mathrm{p}}$ has been previously measured in vivo, however tissue distribution predictive models based on physicochemical and in vitro binding characteristics can effectively replace in vivo models in early-stage drug discovery (Poulin and Theil, 2002; Rodgers and Rowland, 2007; Poulin, 2015). In the CNS, Kp (brain, plasma) does not always correlate to predicted efficacy. Discrepancies are likely due to drug nonspecific binding to proteins in the brain. Brain $f_{\mathrm{u}}$ quantification is paramount and must be used as a correction factor for total brain concentration, therefore improving mechanistic PK/PD evaluations (Read and Braggio, 2010). In vivo validation of brain $f_{\mathrm{u}}$ can be difficult to measure, although drug concentration in the CSF 
has been used as a surrogate method for quantifying brain $f_{\mathrm{u}}$ (Liu et al., 2006; Lin, 2008). While differences between small molecules and ASOs (high molecular weight and polyanionic character) must be taken into consideration, ASO in-vitro protein binding may provide important information on drug potency, distribution, and clearance.

Overall, the ability to modulate protein expression makes ASOs a promising therapeutic modality for the treatment of neurodegenerative diseases as Alzheimer's disease, Parkinson's disease, Huntington's disease, and amyotrophic lateral sclerosis (ALS) have been linked to toxic protein accumulation (Bossy-Wetzel et al., 2004; Smith et al., 2006; DeVos and Miller, 2013). Due to relatively large molecular size and negative charge, ASOs do not efficiently cross the blood brain barrier, but regardless of this limitation, and through novel delivery methods, ASOs continue to undergo clinical trials for CNS related diseases (Hammarlund-Udenaes et al., 2008; Bennett et al., 2019; Tabrizi et al., 2019; Leavitt Blair and Tabrizi Sarah, 2020). With the exciting progress in ASO treatment of neurological diseases, this study provides valuable insight into ASO pharmacokinetics in the brain and CSF and can be an invaluable tool in the support and characterization of in vivo PK-PD data.

\section{Authorship Contributions}

Participated in research design: Guimaraes, Yuan, Li.

Conducted experiments: Guimaraes, $\mathrm{Li}$

Performed data analysis: Guimaraes, $\mathrm{Li}$

Wrote or contributed to the writing of the manuscript: Guimaraes, Yuan, Li.

\section{References}

Alarcón-Arís D, Pavia-Collado R, Miquel-Rio L, Coppola-Segovia V, Ferrés-Coy A, Ruiz-Bronchal E, Galofré M, Paz V, Campa L, Revilla R, Montefeltro A, Kordower JH, Vila M, Artigas F, and Bortolozzi A (2020) Anti- $\alpha$-synuclein ASO delivered to monoamine neurons prevents $\alpha$ - 
synuclein accumulation in a Parkinson's disease-like mouse model and in monkeys. EBioMedicine 59:102944-102944.

Basiri B, Sutton JM, Hooshfar S, Byrnes CC, Murph MM, and Bartlett MG (2019) Direct identification of microribonucleic acid miR-451 from plasma using liquid chromatography mass spectrometry. Journal of Chromatography A 1584:97-105.

Bennett CF, Baker BF, Pham N, Swayze E, and Geary RS (2017) Pharmacology of Antisense Drugs. Annual Review of Pharmacology and Toxicology 57:81-105.

Bennett CF, Krainer AR, and Cleveland DW (2019) Antisense Oligonucleotide Therapies for Neurodegenerative Diseases. Annu Rev Neurosci 42:385-406.

Bossy-Wetzel E, Schwarzenbacher R, and Lipton SA (2004) Molecular pathways to neurodegeneration. Nature Medicine 10:S2-S9.

Cossum PA, Sasmor H, Dellinger D, Truong L, Cummins L, Owens SR, Markham PM, Shea JP, and Crooke S (1993) Disposition of the 14C-labeled phosphorothioate oligonucleotide ISIS 2105 after intravenous administration to rats. Journal of Pharmacology and Experimental Therapeutics 267:1181-1190.

DeVos SL and Miller TM (2013) Antisense oligonucleotides: treating neurodegeneration at the level of RNA. Neurotherapeutics 10:486-497.

Geary RS (2001) Pharmacokinetics of phosphorothioate antisense oligodeoxynucleotides. Curr Opin Investig Drugs 2:562-573.

Geary RS, Norris D, Yu R, and Bennett CF (2015) Pharmacokinetics, biodistribution and cell uptake of antisense oligonucleotides. Advanced Drug Delivery Reviews 87:46-51.

Geary RS, Watanabe TA, Truong L, Freier S, Lesnik EA, Sioufi NB, Sasmor H, Manoharan M, and Levin AA (2001) Pharmacokinetic Properties of 2'-O-(2-Methoxyethyl)-Modified Oligonucleotide Analogs in Rats. Journal of Pharmacology and Experimental Therapeutics 296:890-897.

Hammarlund-Udenaes M, Fridén M, Syvänen S, and Gupta A (2008) On the rate and extent of drug delivery to the brain. Pharm Res 25:1737-1750.

Henry SP, Johnson M, Zanardi TA, Fey R, Auyeung D, Lappin PB, and Levin AA (2012) Renal uptake and tolerability of a 2'-O-methoxyethyl modified antisense oligonucleotide (ISIS 113715) in monkey. Toxicology 301:13-20.

Hirate J, Zhu C, Horikoshi I, and Nagase S (1990) Disposition of warfarin in analbuminemic rats. International Journal of Pharmaceutics 65:149-157.

Hughes TP, Pickels EG, and Horsfall FL (1938) A METHOD FOR DETERMINING THE DIFFERENTIAL SEDIMENTATION OF PROTEINS IN THE HIGH SPEED CONCENTRATION CENTRIFUGE. J EXP Med 67:941-952.

Humphreys SC, Thayer MB, Lade JM, Wu B, Sham K, Basiri B, Hao Y, Huang X, Smith R, and Rock BM (2019) Plasma and Liver Protein Binding of N-Acetylgalactosamine-Conjugated Small Interfering RNA. Drug Metabolism and Disposition 47:1174.

Isbell J, Yuan D, Torrao L, Gatlik E, Hoffmann L, and Wipfli P (2019) Plasma Protein Binding of Highly Bound Drugs Determined With Equilibrium Gel Filtration of Nonradiolabeled Compounds and LC-MS/MS Detection. Journal of Pharmaceutical Sciences 108:10531060. 
Jones $\mathrm{H}$ and Rowland-Yeo K (2013) Basic concepts in physiologically based pharmacokinetic modeling in drug discovery and development. CPT Pharmacometrics Syst Pharmacol 2:e63-e63.

Kalvass JC and Maurer TS (2002) Influence of nonspecific brain and plasma binding on CNS exposure: implications for rational drug discovery. Biopharmaceutics \& Drug Disposition 23:327-338.

Kalvass JC, Maurer TS, and Pollack GM (2007) Use of Plasma and Brain Unbound Fractions to Assess the Extent of Brain Distribution of 34 Drugs: Comparison of Unbound Concentration Ratios to in Vivo P-Glycoprotein Efflux Ratios. Drug Metabolism and Disposition 35:660-666.

Leavitt Blair R and Tabrizi Sarah J (2020) Antisense oligonucleotides for neurodegeneration. Science 367:1428-1429.

Levin AA, Yu RZ, and Geary RS (2007) Basic Principles of the Pharmacokinetics of Antisense Oligonucleotide Drugs, in: Antisense Drug Technology (Crooke ST ed), pp 183-216, CRC Press.

Li P, Gong Y, Kim J, Liu X, Gilbert J, Kerns HM, Groth R, and Rooney M (2020) Hybridization Liquid Chromatography-Tandem Mass Spectrometry: An Alternative Bioanalytical Method for Antisense Oligonucleotide Quantitation in Plasma and Tissue Samples. Analytical Chemistry 92:10548-10559.

Lin JH (2008) CSF as a surrogate for assessing CNS exposure: an industrial perspective. Curr Drug Metab 9:46-59.

Liu X, Smith BJ, Chen C, Callegari E, Becker SL, Chen X, Cianfrogna J, Doran AC, Doran SD, Gibbs JP, Hosea N, Liu J, Nelson FR, Szewc MA, and Van Deusen J (2006) Evaluation of Cerebrospinal Fluid Concentration and Plasma Free Concentration As a Surrogate Measurement for Brain Free Concentration. Drug Metabolism and Disposition 34:1443.

Liu X, Wright M, and Hop CECA (2014) Rational Use of Plasma Protein and Tissue Binding Data in Drug Design. Journal of Medicinal Chemistry 57:8238-8248.

Mariappan TT, Sandhya M, and Punit M (2013) Insight into Tissue Unbound Concentration: Utility in Drug Discovery and Development. Current Drug Metabolism 14:324-340.

Mazur C, Powers B, Zasadny K, Sullivan JM, Dimant H, Kamme F, Hesterman J, Matson J, Oestergaard M, Seaman M, Holt RW, Qutaish M, Polyak I, Coelho R, Gottumukkala V, Gaut CM, Berridge M, Albargothy NJ, Kelly L, Carare RO, Hoppin J, Kordasiewicz H, Swayze EE, and Verma A (2019) Brain pharmacology of intrathecal antisense oligonucleotides revealed through multimodal imaging. JCI Insight 4:e129240.

Miller TM, Pestronk A, David W, Rothstein J, Simpson E, Appel SH, Andres PL, Mahoney K, Allred P, Alexander K, Ostrow LW, Schoenfeld D, Macklin EA, Norris DA, Manousakis G, Crisp M, Smith R, Bennett CF, Bishop KM, and Cudkowicz ME (2013) An antisense oligonucleotide against SOD1 delivered intrathecally for patients with SOD1 familial amyotrophic lateral sclerosis: a phase 1, randomised, first-in-man study. The Lancet Neurology 12:435-442.

Min HS, Kim HJ, Naito M, Ogura S, Toh K, Hayashi K, Kim BS, Fukushima S, Anraku Y, Miyata K, and Kataoka K (2020) Systemic Brain Delivery of Antisense Oligonucleotides across the Blood-Brain Barrier with a Glucose-Coated Polymeric Nanocarrier. Angew Chem Int Ed Engl 59:8173-8180. 
Pacifici GM and Viani A (1992) Methods of Determining Plasma and Tissue Binding of Drugs. Clinical Pharmacokinetics 23:449-468.

Poulin P (2015) Drug Distribution to Human Tissues: Prediction and Examination of the Basic Assumption in <em $>$ In Vivo</em> Pharmacokinetics-Pharmacodynamics (PK/PD) Research. Journal of Pharmaceutical Sciences 104:2110-2118.

Poulin P and Theil FP (2002) Prediction of Pharmacokinetics Prior to <em $>$ In Vivo $</$ em $>$ Studies.

1. Mechanism\&\#x2010;Based Prediction of Volume of Distribution. Journal of Pharmaceutical Sciences 91:129-156.

Read KD and Braggio S (2010) Assessing brain free fraction in early drug discovery. Expert Opinion on Drug Metabolism \& Toxicology 6:337-344.

Rinaldi C and Wood MJA (2018) Antisense oligonucleotides: the next frontier for treatment of neurological disorders. Nature Reviews Neurology 14:9-21.

Roberts JA, Pea F, and Lipman J (2013) The Clinical Relevance of Plasma Protein Binding Changes. Clinical Pharmacokinetics 52:1-8.

Rocca C, Dennin S, Gu Y, Kim J, Chigas S, Najarian D, Chong S, Gutierrez S, Butler J, Charisse K, Robbie G, Xu Y, and Brown K (2019) Evaluation of electrophoretic mobility shift assay as a method to determine plasma protein binding of siRNA. Bioanalysis 11:1927-1939.

Rodgers T and Rowland M (2007) Mechanistic Approaches to Volume of Distribution Predictions: Understanding the Processes. Pharm Res 24:918-933.

Ross SM (2003) Peirce's Criterion for the Elimination of Suspect Experimental Data. Journal of Engineering Technology 20:38-41.

Rowland M, Tozer TN, and Rowland M (2011) Clinical pharmacokinetics and pharmacodynamics : concepts and applications. Wolters Kluwer Health/Lippincott William \& Wilkins, Philadelphia.

Smith RA, Miller TM, Yamanaka K, Monia BP, Condon TP, Hung G, Lobsiger CS, Ward CM, McAlonis-Downes M, Wei H, Wancewicz EV, Bennett CF, and Cleveland DW (2006) Antisense oligonucleotide therapy for neurodegenerative disease. J Clin Invest 116:2290-2296.

Srinivasan SK, Tewary HK, and Iversen PL (1995) Characterization of Binding Sites, Extent of Binding, and Drug Interactions of Oligonucleotides with Albumin. Antisense Research and Development 5:131-139.

Stein CA and Castanotto D (2017) FDA-Approved Oligonucleotide Therapies in 2017. Mol Ther 25:1069-1075.

Tabrizi SJ, Leavitt BR, Landwehrmeyer GB, Wild EJ, Saft C, Barker RA, Blair NF, Craufurd D, Priller J, Rickards H, Rosser A, Kordasiewicz HB, Czech C, Swayze EE, Norris DA, Baumann T, Gerlach I, Schobel SA, Paz E, Smith AV, Bennett CF, and Lane RM (2019) Targeting Huntingtin Expression in Patients with Huntington's Disease. New England Journal of Medicine 380:2307-2316.

Toma C-M, Imre S, Vari C-E, Muntean D-L, and Tero-Vescan A (2021) Ultrafiltration Method for Plasma Protein Binding Studies and Its Limitations. Processes 9:382.

Trainor GL (2007) The importance of plasma protein binding in drug discovery. Expert Opinion on Drug Discovery 2:51-64.

Wang L, Meng M, and Reuschel S (2013) Regulated bioanalysis of oligonucleotide therapeutics and biomarkers: qPCR versus chromatographic assays. Bioanalysis 5:2747-2751. 
Watanabe TA, Geary RS, and Levin AA (2006) Plasma Protein Binding of an Antisense Oligonucleotide Targeting Human ICAM-1 (ISIS 2302). Oligonucleotides 16:169-180.

Wurster CD and Ludolph AC (2018) Antisense oligonucleotides in neurological disorders. Ther Adv Neurol Disord 11:1756286418776932-1756286418776932.

Zhang F, Xue J, Shao J, and Jia L (2012) Compilation of 222 drugs' plasma protein binding data and guidance for study designs. Drug Discovery Today 17:475-485.

Footnotes:

This work received no external funding. No author has an actual or perceived conflict of interest with the contents of this article. 


\section{Figure Legends}

Figure 1. A) Recovery of $1 \mu \mathrm{M}$ ASO-1 in PBS through different filter pre-treatments. B) Recovery of $1 \mu \mathrm{M}$ ASO-1 in different MWCO filter sizes pre-treated with $0.1 \%$ Tween-20 (PBST). C) Recovery of $1 \mu \mathrm{M}$ warfarin and antipyrine in different MWCO filter sizes pre-treated with $0.1 \%$ Tween-20 (PBST).

Figure 2. A) ASO-1 plasma $f u$ in different MWCO filter sizes. B) ASO-1, ASO-2 and ASO-3 plasma $f \mathrm{u}$ in $30 \mathrm{~K}$ MWCO filters.

Figure 3. ASO brain $f \mathrm{u}$ across different filter sizes.

Figure 4. A) ASO-1 drug unbound fraction in CSF and artificial CSF. B) ASO CSF unbound fraction in 50K MWCO filters.

Figure 5. Rapid equilibrium dialysis (RED) system equilibrium through different membrane pretreatments. 


\section{Tables}

Table 1. ASO Analytes and Internal Standard

\begin{tabular}{lllll}
\hline Name & MW & Sequence & Chemistry $^{*}$ & Use \\
& $(\mathrm{kDa})$ & length & & \\
\hline ASO-1 & 7.1 & 20 & $5-10-5$ MOE-gapmer & analyte \\
ASO-2 & 7.1 & 20 & $5-10-5$ MOE-gapmer & analyte \\
ASO-3 & 5.9 & 17 & MOE/cEt-gapmer & analyte \\
ASO-4 & 7.9 & 20 & uniform MOE with PS backbone & internal standard \\
$*$ MOE, 2'-O-(2-methoxyethyl)-oligoribonucleotides; cEt, constrained & ethyl motif; PS, \\
phosphorothioate linkage.
\end{tabular}


Table 2. Orthogonal validation of $30 \mathrm{kDa}$ MWCO ultrafiltration method through previously characterized small molecules.

\begin{tabular}{|c|c|c|c|}
\hline & $\begin{array}{l}\text { Experimental } \\
\text { Values }(\%)\end{array}$ & $\begin{array}{l}\text { Experimental Values } \\
\text { Generated Using a Standard } \\
\text { RED Protocol }(\%)\end{array}$ & $\begin{array}{l}\text { Literature } \\
\text { Values }(\%)\end{array}$ \\
\hline & \multicolumn{3}{|l|}{ Plasma } \\
\hline \multicolumn{4}{|c|}{ Warfarin } \\
\hline Mouse & $5.5 \pm 0.3$ & $4.3 \pm 0.9$ & $4.6 \pm 0.4^{\mathrm{a}}$ \\
\hline Rat & $1.0 \pm 0.5$ & $0.8 \pm 0.4$ & $1.1^{\mathrm{b}}$ \\
\hline Dog & $3.2 \pm 0.1$ & $3.9 \pm 0.7$ & $4.0 \pm 0.1^{\mathrm{a}}$ \\
\hline Human & $1.1 \pm 0.5$ & $1.5 \pm 0.3$ & $1.0^{\mathrm{c}}$ \\
\hline Monkey & $0.8 \pm 0.2$ & $1.0 \pm 0.7$ & $0.7 \pm 0.1^{\mathrm{a}}$ \\
\hline \multicolumn{4}{|c|}{ Antipyrine } \\
\hline \multirow[t]{2}{*}{ Human } & $92.8 \pm 2.2$ & & $90^{\mathrm{d}}$ \\
\hline & Brain & & \\
\hline \multicolumn{4}{|c|}{ Verapamil } \\
\hline Rat & $2.8 \pm 0.3$ & $3.8 \pm 1.4$ & $3.3 \pm 2.0^{\mathrm{e}}$ \\
\hline
\end{tabular}


Table 3: Ultracentrifugation plasma $f_{\mathrm{u}}$ and recovery in PBS values for ASO-1 and warfarin $(\mathrm{n}=$ 1)

\begin{tabular}{|c|c|c|c|c|c|c|c|c|}
\hline \multicolumn{9}{|c|}{ Plasma $f_{\mathrm{u}_{-}}(\%)$} \\
\hline & \multicolumn{4}{|c|}{ ASO-1 } & \multicolumn{4}{|c|}{ Warfarin } \\
\hline & 1 hour & 2 hours & 3 hours & 4 hours & 1 hour & 2 hours & 3 hours & 4 hours \\
\hline Q1 & 14.3 & 3.4 & 2.6 & 2.0 & 15.7 & 0.5 & 0.6 & 0.6 \\
\hline Q2 & 63.0 & 10.7 & 3.8 & 2.9 & 90.8 & 6.2 & 0.7 & 0.5 \\
\hline Q3 & 122.7 & 160.7 & 55.4 & 58.6 & 150.0 & 195.7 & 71.1 & 82.2 \\
\hline Q4 & 204.8 & 244.5 & NA & NA & 169.2 & 184.4 & NA & NA \\
\hline \multirow[t]{4}{*}{$\begin{array}{l}\text { Total } \\
\text { Recovery } \\
\end{array}$} & 101.2 & 104.8 & NA & NA & 106.4 & 96.7 & NA & NA \\
\hline & \multicolumn{8}{|c|}{ Recovery in PBS (\%) } \\
\hline & \multicolumn{4}{|c|}{ ASO-1 } & \multicolumn{4}{|c|}{ Warfarin } \\
\hline & 1 hour & 2 hours & 3 hours & 4 hours & 1 hour & 2 hours & 3 hours & 4 hours \\
\hline Q1 & 74.3 & 75.2 & 78.5 & 82.5 & 62.5 & 73.0 & 109.1 & 106.1 \\
\hline Q2 & 97.9 & 77.7 & 79.6 & 80.1 & 77.8 & 72.4 & 107.5 & 104.0 \\
\hline Q3 & 87.7 & 85.9 & 84.1 & 101.2 & 79.0 & 76.1 & 111.9 & 107.8 \\
\hline Q4 & 153.5 & 152.0 & 110.9 & 129.5 & 210.2 & 216.8 & 107.5 & 119.5 \\
\hline $\begin{array}{l}\text { Total } \\
\text { Recovery }\end{array}$ & 103.4 & 97.7 & 88.3 & 98.3 & 107.4 & 109.6 & 109.0 & 109.4 \\
\hline
\end{tabular}

NA: recovery was unmeasurable due to solidified Q4 matrix after ultracentrifugation 
DMD Fast Forward. Published on December 17, 2021 as DOI: 10.1124/dmd.121.000751

This article has not been copyedited and formatted. The final version may differ from this version.

\section{Figures}


Figure 1

A)

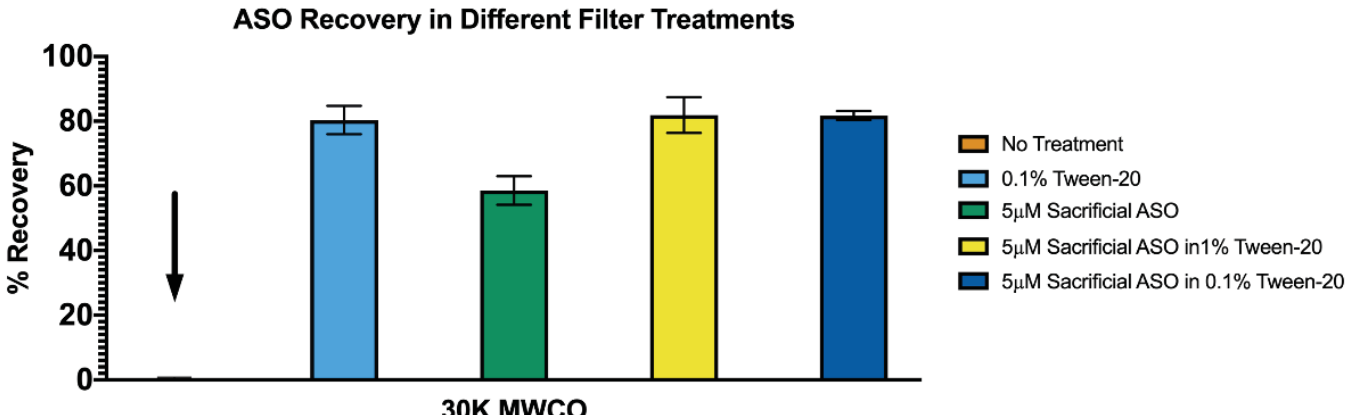

B)

ASO Recovery in $0.1 \%$ Tween-20 Treated Filters

C)

Small Molecule Recovery in 0.1\% Tween-20 Treated Filters
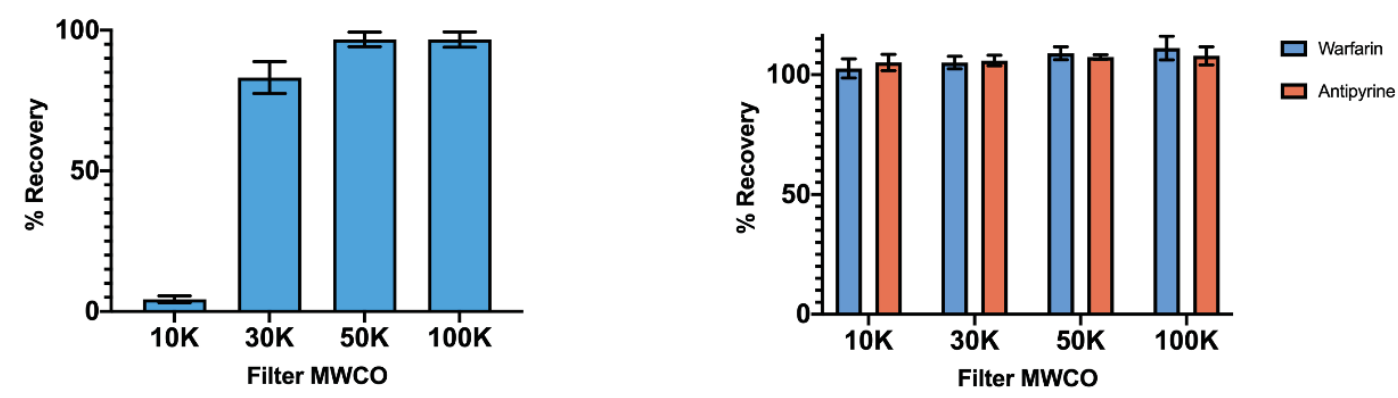
Figure 2

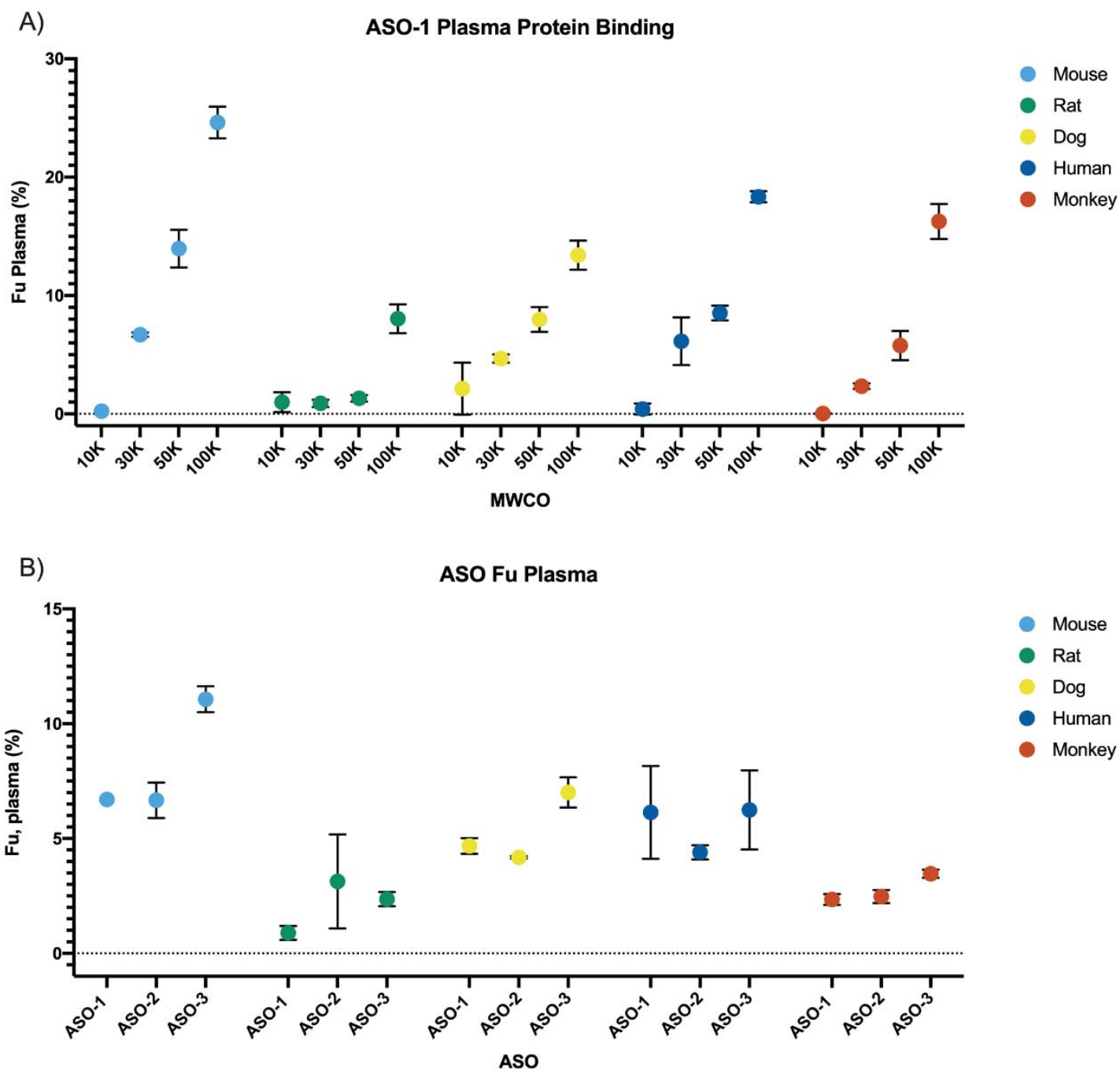


Figure 3

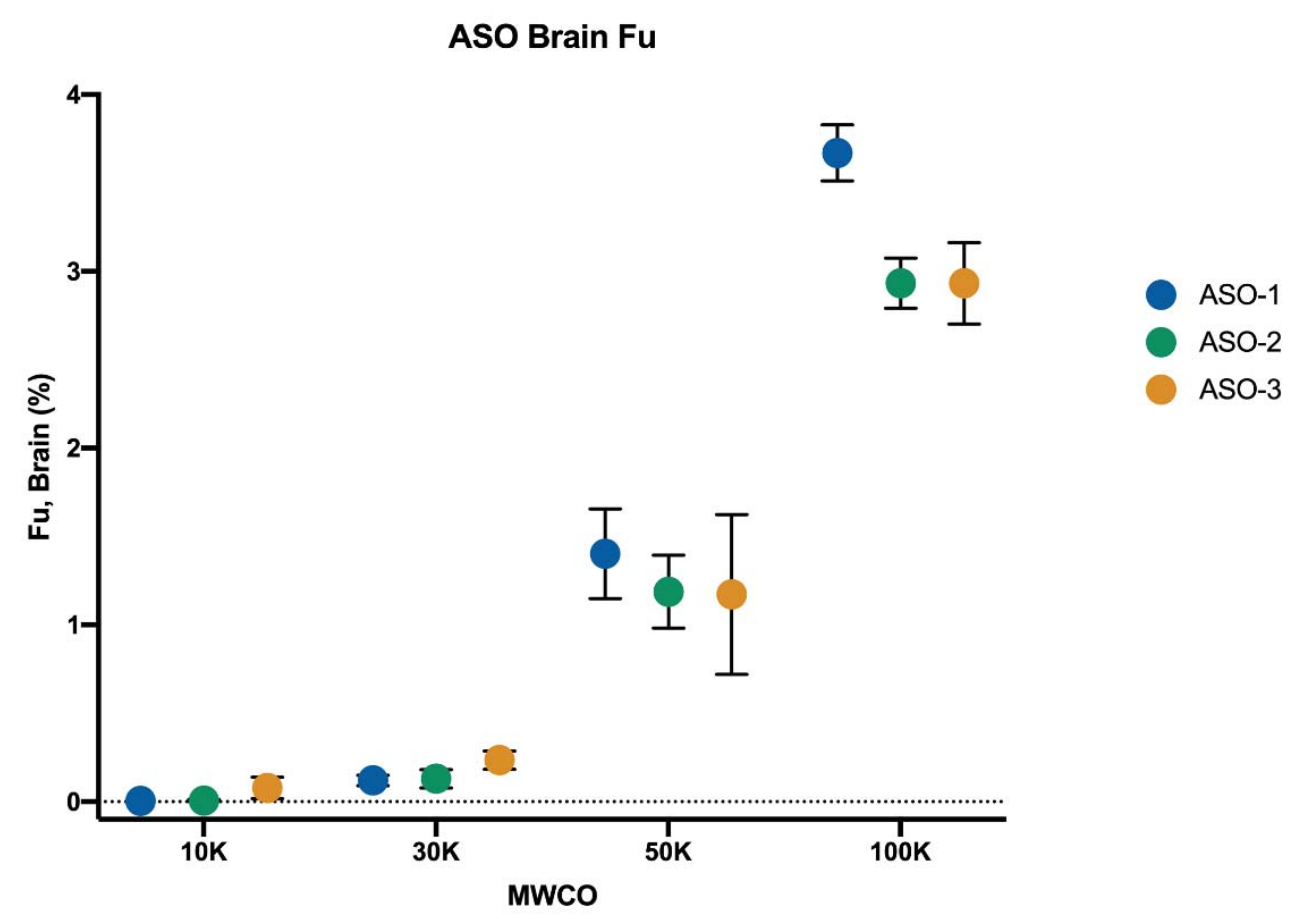


Figure 4

A) ASO Free Fraction in CSF and ACSF

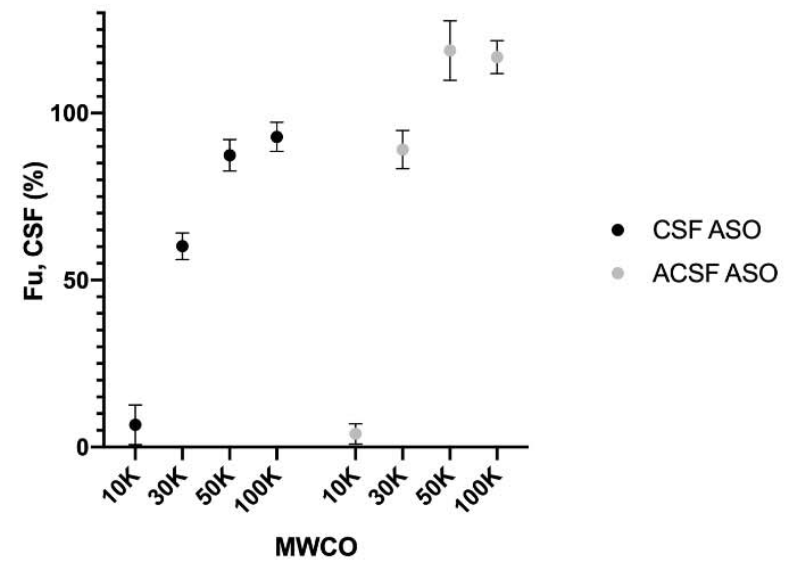

B) ASO Fu Cerebral Spinal Fluid

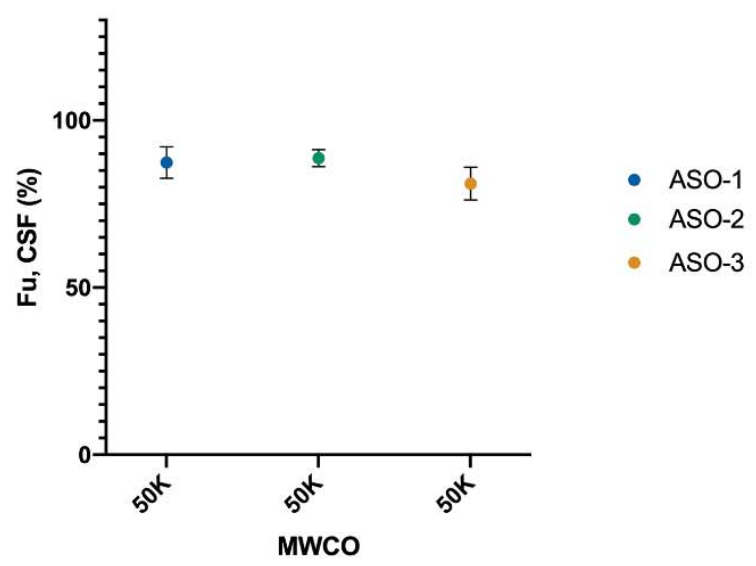


Figure 5

RED Equilibrium

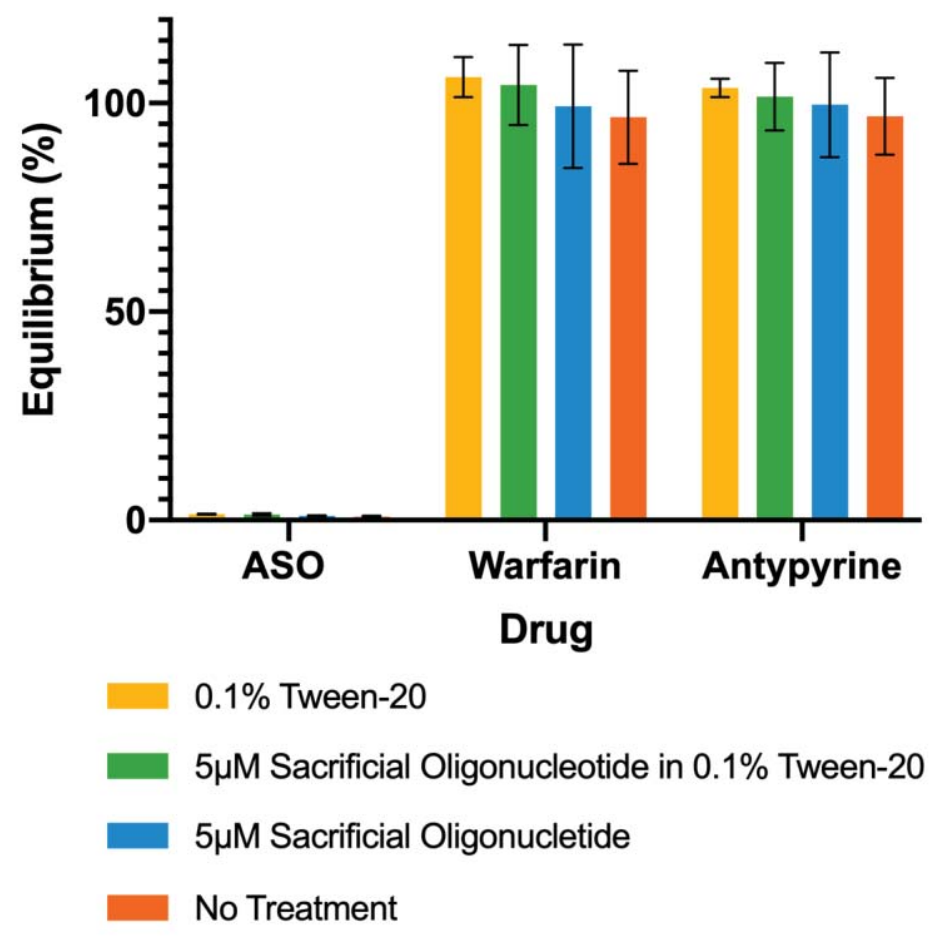

